\title{
Heros Be Star Campaigns
}

\author{
S. Štefl
}

Astronomical Institute, CZ-251 65 Ondřejov, Czech Republic

\section{T. Rivinius}

\section{Landessternwarte Königstuhl, D-69117 Heidelberg, Germany}

\begin{abstract}
Systematic Be star observations with the HeRos spectrograph have been carried out in several long observing runs and seasons, mainly at the ESO La Silla Observatory. The HEROS data represents one of the most extended spectroscopic data sets for the Be star research. More complete and efficient diagnostics and a higher frequency resolution in analyses of periodic spectral variations were achieved, thanks to a combination of relatively high spectral resolution, a large sample of monitored spectral lines of different formation properties, long observing runs, and a relatively high frequency of observations of main targets.

Important results concerning different parts of the extended atmospheres of Be stars and having strong impact on our understanding of these objects have been achieved.
\end{abstract}

\section{Introduction}

Heidelberg Extended Range Optical Spectrograph (HERos) is a fiber fed transportable spectrograph with spectral resolving power of 20000 over the spectral range $3450-8620 \AA$. The Heros optical scheme is shown in Figure 1. The blue and red spectrograph channels have separate cameras and detectors and are divided by a gap of about $200 \AA$ wide centered at $\lambda \approx 5750 \AA$.

HERos has been used for several campaigns focused on different types of hot stars, e.g. luminous blue variables, OBA super- and hypergiants, $W R+O$ binaries and Be stars. Observations at the La Silla observatory were involved also in the MUSICOS 98 south campaign. Kaufer (1998) gives technical details of the instrument and an overview over some non-Be related Heros projects.

In the $\mathrm{Be}$ star campaigns, more than 2000 spectra were obtained between 1992 and 1999. In most of the observations, HEROS was attached to the ESO 0.5 and $1.5 \mathrm{~m}$ telescopes of the ESO La Silla Observatory. The 2.2 and $1.2 \mathrm{~m}$ telescopes at Calar Alto, the Heidelberg $0.7 \mathrm{~m}$ Waltz telescope, and the Tautenburg $2 \mathrm{~m}$ telescope were used for the remaining observations. Some of the most recent observations in some projects reported below were carried out with the FEROS spectrograph attached to the ESO $1.5 \mathrm{~m}$ telescope. FEROS represents a continuation of the Heidelberg program to an instrument with higher spectral 


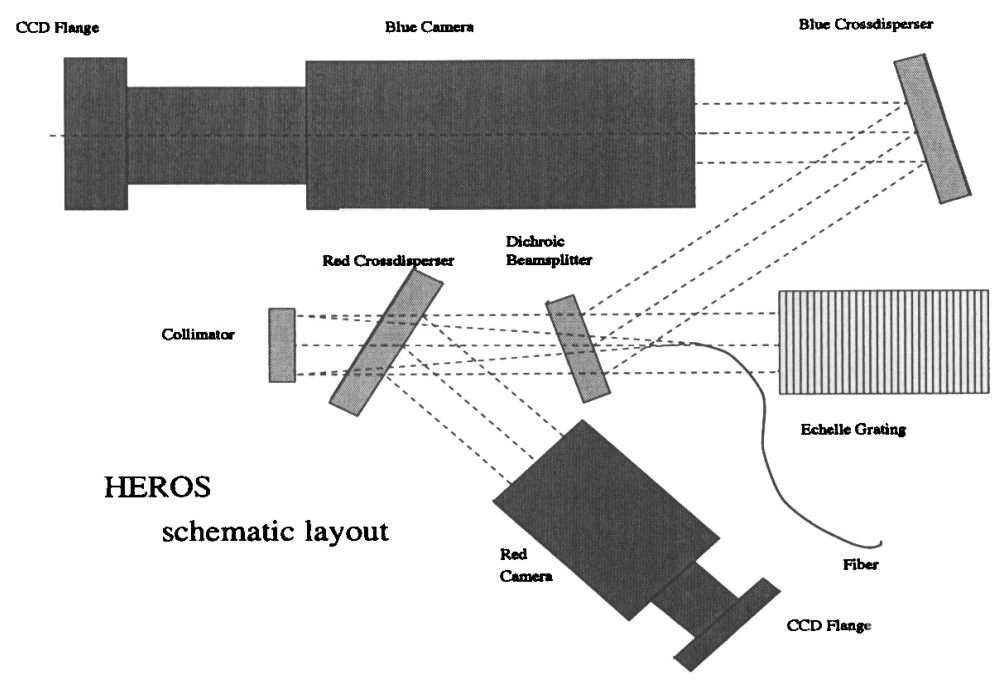

Figure 1. Optical scheme of the HERos spectrograph (courtesy A. Kaufer)

resolution (48000) and higher $S / N$. Such an improvement permits finer physical details to be observed and modeled.

We may emphasize two characteristic features of the HEROs Be star observations, which enabled us to make significant progress in the field, although the program was carried out by only a relatively small group (see acknowledgments) and only with small to medium-sized telescopes:

- spectra with high resolution over more than the entire optical range were used for multi-line analysis (25-30 lines). This way we could reach a higher significance of the results and study relations between photospheric and circumstellar phenomena,

- combining long observing runs (typically 1-3.5 months) from several seasons for every selected target, we could obtain hitherto hardly achievable frequency resolution in analyses of periodic variations and study phenomena variable on time scales of months-years. Thanks to relatively high temporal resolution (typically 1 spectrum per night, but as many as 5-6 spectra per night for a few stars in some runs), we could also study relations between rapid and long-term variations and the long-term development of the rapid variability.

With its broad spectral coverage, HERos could monitor spectral lines coming from different parts of the Be star atmospheres and circumstellar disks. Analyzing them, we could address a broad range of problems concerning $\mathrm{Be}$ stars, such as rapid line profile variations $(l p v)$, modeling of spectral profiles due to non-radial pulsations $(n r p)$, the nature of Be star outbursts and the structure of the circumstellar disks. We will follow the path from the stellar photosphere through the analysis in the following brief overview of the main results. 


\section{Rapid line profile variations}

\subsection{Multiperiodicity}

Multiperiodicity in rapid $l p v$ can be used as an important criterion in testing of models of the $l p v$. A reliable proof means interpretational problems for all models based on rotational modulation and strong support for the $n r p$ model. For such a test, a few selected stars were monitored during several seasons.

The results published for $\mu$ Cen (Rivinius et al., 1998b) represent the most reliable evidence of spectroscopic multiperiodic variations published for a $\mathrm{Be}$ star up to now. Six periods in two groups around 0.5 and $0.28 \mathrm{~d}$ were detected. Their separation within each group is 0.01-0.02 d. A more detailed discussion of this result can be found in Baade et al. (these proc.). $28 \mathrm{Cyg}$ is a second star with multiperiodic line profile variations in the HERos database. The detected periods are 0.647 and $0.625 \mathrm{~d}$ (see Tubbesing et al., these proc.).

The above results for $\mu$ Cen and 28 Cyg show that the frequency resolution necessary for detections of multiperiodicity may have to be $0.02 \mathrm{c} / \mathrm{d}$ or even better. Multiperiodic variations in $\mu$ Cen and 28 Cyg indicate that at least in some Be stars the line profile variations can be hardly explained by rotational modulation.

\subsection{Modeling of line profile variations}

Using the $n r p$ modeling code by Townsend (1997), variations of spectral lines formed under different physical conditions could be modeled simultaneously. The code enables an adequate treatment of the important role of the equatorto-pole variation of $T_{\text {eff }}$ and $\log g$ due to rapid rotation and the inclusion of realistic limb darkening. The observed and calculated line profiles agree even in details such as spikes and ramps (for their description see e.g. Rivinius et al., $1998 \mathrm{~b})$. The performed modeling supports the $n r p$ interpretation of rapid line profile variations at least in some Be stars. For more detailed results on $\mu$ Cen and $28 \mathrm{CMa}$ see the contributions by Rivinius and Maintz et al., respectively, in these proceedings.

\section{Nature of the Be star outbursts}

\subsection{General phases of the emission outbursts}

The detailed analysis of 11 emission outbursts monitored in $\mu$ Cen led to a general picture of the emission outbursts and definition of their four main phases (Rivinius et al., 1998a). Observations of less frequent outbursts in $\eta \mathrm{Cen}, 28 \mathrm{CMa}$ and 28 Cyg agree well with the generalized scheme shown in Figure 2. The most conspicuous spectral changes appear in the precursor phase. It is characterized by a sudden drop and subsequent recovery of line emission, increased emission in the $\mathrm{HI}$ line wings and a discontinuous increase of the separation of the violet and red peaks in double-peak emission lines. In many outbursts, rapid $V / R$ variations start also in the precursor phase and persist with decreasing amplitude through the next stage of the outburst, when the line emission reaches a maximum, whereas the emission in the wings and the peak separation are decreasing. Also variations in the base width of emission lines from ions like $\mathrm{Fe}^{+}$, 


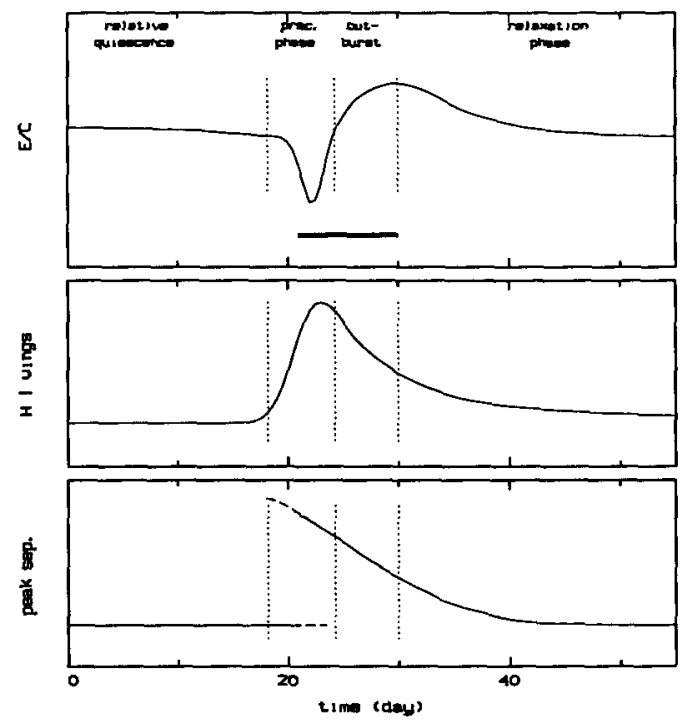

Figure 2. Schematic picture of Be star emission outbursts with four typical main phases. The bold line in the upper panel indicates the occurrence of temporary rapid $V / R$ variations and transient periods. From Rivinius et al. (1998a).

which are kinematically dominated up to the outer line wings, were detected (e.g., Fig. 1 of Tubbesing et al., these proc.). Since the base width is indicative of the dimension of the inner edge of the disk, there seem to be times, when the disk is not reaching down to the star. This is supported by observations in which after a couple of weeks to months high excitation emission lines may have already disappeared (e.g., the upper lines of the Paschen series), while low excitation lines (e.g., lower Balmer lines such as $\mathrm{H} \alpha$ ) are still at peak strength. All spectral features return asymptotically to their original values during the final relaxation phase. Fig. 2 shows typical time scales of individual phases for $\mu \mathrm{Cen}$, but the time scales can be 5-10 times longer in other stars, e.g. in $28 \mathrm{CMa}$.

\subsection{Empirical ephemeris of the emission outbursts of $\mu$ Cen}

The monitoring of $\mu$ Cen revealed correlations between rapid variations of the photospheric absorption profiles and spectral signatures used as indicators of emission outbursts. The vector fields of all four periods in the $0.5 \mathrm{~d}$ group have homologous topology and the vector sum of $\mathcal{P}_{1} / \mathcal{P}_{2} / \mathcal{P}_{3}$ is largest at the times of outbursts. Due to its relative weakness $\mathcal{P}_{4}$ was neglected.

This fact was used for the construction of the empirical ephemeris. When the sum of the reconstructed radial velocity variations has a maximal amplitude, an outburst is to be expected. This ephemeris was successful in predicting the maximal amplitude in our own data and those of Hanuschik et al. (1993) to within 1 week. Outbursts predicted for 1997 and 1999, too, were detected in spectra taken at the expected epochs. The line profile and emission varia- 
tions of 28 Cyg seem to permit the construction of a similar outburst ephemeris (Tubbesing et al., these proc.)

\section{Emission disks}

\subsection{Transient periodicities}

Secondary periodic variations of temporary character were discovered in line profile variations of $\mu \mathrm{Cen}, \eta \mathrm{Cen}$, and $28 \mathrm{CMa}$ and reported under the term "transient periodicities" (Štefl et al., 1998; Stefl et al., these proc.). These variations co-exist with the photospheric line-profile variations, but their periods differ typically by $10 \%$. Generally, they appear after the outbursts and are present for a few days $(\mu \mathrm{Cen})$ up to a month $(28 \mathrm{CMa})$. They can be detected in line profile variations of most absorption lines (mainly in their wings), in modes of $\mathrm{HI}$ absorption lines, and in the $V / R$ ratio and modes of emission lines. They are either not at all or only weakly detectable in photospheric lines and attain significant strengths only in lines formed above the photosphere (as emission components). When these periodicities re-appear, the period is not the same as at the previous epoch. However, in $\mu \mathrm{Cen}$, the transient periodicities excited during different outbursts fall within an interval of $\pm 10 \%$ of one another. So far, no more than one transient period has been detected at a given time. The present data coverage and sampling are not sufficient to answer, if the variability stops abruptly or drops below the detection threshold gradually. However, the process might be connected to the "damped ringing" observed in the UV and optical photometry.

Due to the existence of transient periodicities, the standardly measured spectral parameters may show different periodicity patterns in different time intervals. For example, modes of $\mathrm{H} \mathrm{I}$ (and line profile variations) can temporarily have a biperiodic nature and, therefore, a different variability pattern than modes of He I lines. This implies a general conclusion for time series analyses of Be stars: One should avoid combining modes of $H e \mathrm{I}$ and $H \mathrm{I}$ lines unless the periodicity character can be checked independently for all data subsets. Combining the data from intervals with different periodicity patterns, a false multiperiodicity or varying periods can be derived. The same problem concerns $V / R$ ratios and modes of emission lines.

HEROS spectra showed also high-velocity absorption components (hva). As with the transient periods, the hva may occur in the precursor or early outburst phase. Their RVs are more negative than $v \sin i$ and appear in all stronger $\mathrm{He} I$ lines. Smith (these proc.) models the hva and apparent accompanying emission in HERos spectra of $\mu$ Cen and concludes that they are formed in the outflowing, perhaps magnetically confined gas during the outburst. It is very probable that both the hva and the transient periods are phenomena closely related to the outburst mechanism and provide information about it. Their mutual relation has up to now not been studied.

\subsection{Central quasi-emission bumps}

Central quasi-emission bumps (CQE's), illustrated in Fig. 3, were observed in $\epsilon$ Cap, $\eta$ Cen, $\omega$ Car, $\nu$ Pup, 4 Her and $o$ And. The only previously pub- 


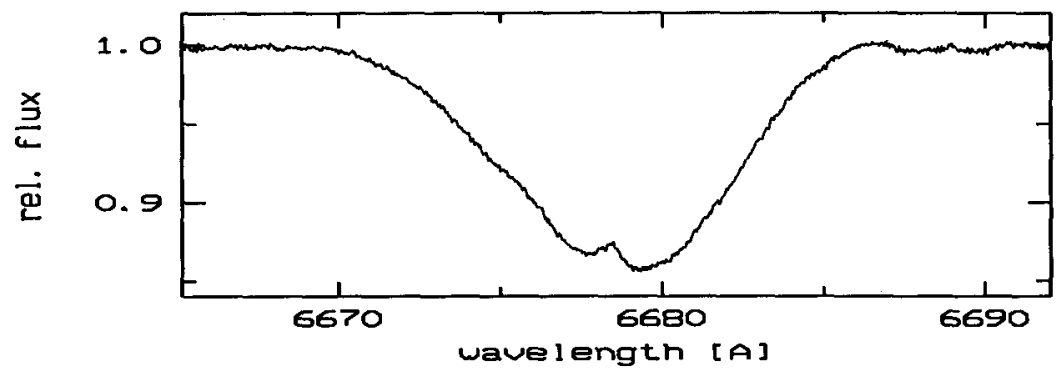

Figure 3. Central quasi-emission bump in $\epsilon$ Cap, He I 6678 observed with the ESO Coudé Auxiliary Telescope and Coudé Echelle Spectrometer in November, 1994

lished explanations of these small features assumed a photospheric origin. Baade (1990) and Jeffery (1991) interpreted these spectral features as a consequence of the equator-to-pole variation of effective gravity and temperature, while Zorec (1994) attributed it to differential rotation.

The systematic HEROS monitoring of all these stars resulted in the formulation of general conditions under which CQE's appear and consequently in an explanation of their origin (Rivinius et al, 1999). CQE's appear if/when:

- the star possesses a circumstellar disk,

- the disk is seen edge-on,

- the continuum opacity of the disk is low,

- the outer radius of the disk is small,

- the broadening of the shell lines is low.

A qualitative analysis of HEROS observations yielded convincing evidence that CQE's are formed in the circumstellar disks of Be stars. Their observed properties agree well with the features predicted in Hanuschik's (1995) calculations of the iso-radial velocity contours in the disk. The CQE's are not related to any emission process but represent an absorption minimum in the circumstellar rotating disk. They result from the local minimum at zero radial velocity in the fraction of the stellar disk that is occulted by the circumstellar gas moving perpendicular to the line of sight.

CQE's offer a great potential for studies of the dynamics and structure of the disks with the help of quantitative tomography. The agreement of the observations with Hanuschik's model supports the Keplerian rotation of the disk and limits the applicability of the wind-compressed model by Bjorkman and Cassinelli (1993).

\subsection{Structure of the emission disks of $\phi$ Per and 59 Cyg}

$\phi$ Per is a binary system with a period of $126 \mathrm{~d}$, consisting of a B2Ve star and a hot O-type companion. Emission components, variations of which follow the orbital period, belong to the until recently unexplained puzzles of the system. Multi-line analyses focussed on the Fe II and He I emission lines and based partly 
on the Heros data helped to formulate detailed properties, an explanation and a model of the $\mathrm{HeI}$ emission lines, and the discovery of long-term variations of FeII and HeI emission lines (Štefl et al., 2000; Hummel et al., these proc.; Hummel \& Štefl, in preparation).

Emission components were discovered in 3 more He I lines. They occur only in lines coming from transitions between the second and third He I atomic levels, while the shell component without emission appears only in triplet lines excited from the $2^{3} P^{0}$ level. The derived properties of the HeI emission components indicate that they are formed in the circumprimary disk. The orbital variations of $\mathrm{He}$ I 6678 can be fitted by a model, in which the emission component is formed in a sector of the circumprimary disk, which is excited by the secondary $\mathrm{O}$ star. By fitting the calculated emission profiles to the observed ones in 20 phase bins, the geometrical parameters of the sector were derived.

Changes in the symmetry of Fe II and HeI lines after 1997 indicate that a new global density pattern was formed in the inner region of the disks.

$\phi$ Per may not be exceptional but rather a prototype of binary systems with anisotropically excited circumprimary disks. HERos observations of the Be star $59 \mathrm{Cyg}$ revealed an orbital period of $(28.1702 \pm 0.0014) \mathrm{d}$. Many analogies with $\phi$ Per indicate that $59 \mathrm{Cyg}$ is a very similar binary system but viewed at a lower inclination angle (Rivinius \& Štefl, these proc.).

\section{Summary - main achievements of the HEROS project}

Studies based on HERos campaigns touched several key problems of the current Be star research. The main contributions can be generalized as follows:

- a considerable fraction of Be stars in our sample pulsates non-radially. Observations of other Be stars show that the occurrence of spikes is a common phenomenon among low $v \sin i$ Be stars (HR 7316, HR 8520, HR 4074, and to lesser extent also FW CMa, HR 5223, and HR 3642). Since no mechanism other than pulsational $\vartheta$-velocity fields together with a low inclination can up to now explain such spikes, the observations suggest that pulsation is the most common cause for the observed periodic lpv of Be stars,

- for those Be stars with multiperiodic pulsation in closely spaced multiplets with identical values of $\ell$ and $m$, a relation between rapid periodic line profile variations and emission outbursts can be established,

- transient periodicities and high velocity absorption components may hold the key to the understanding of the star-to-disk mass transfer mechanism,

- the disks are at least partly replenished by line emission outbursts,

- the disk rotation is nearly Keplerian, with outflow velocities of the order of a few $\mathrm{km} / \mathrm{s}$ only.

From the HERos campaigns a scenario emerges in which many early-type Be stars pulsate. For at least one (and probably a second) of them, the pulsation is directly connected to the mass transfer to a nearly Keplerian disk via outbursts. The evolution of the disk after an outburst seems to produce a lower density region directly above the star, kind of a central "hole" of the disk. 
Acknowledgments. This review is given on behalf of the entire HeRos group, in which we are pleased to work with D. Baade, A. Kaufer, O. Stahl and B. Wolf, in recent months also W. Hummel, M. Meintz, A. Budovičova and S. Tubbesing. The group partly overlaps with the Heidelberg hot star department led by B. Wolf, which built the HEROs spectrograph. Most of the observing campaigns were prepared and the associated studies carried out with a significant and irreplaceable contribution of D. Baade, whom and M. Smith we also thank for constructive comments on the manuscript of this paper. We also thank the other Heros observers: C. Gummersbach, T. Dumm, T. Gäng, J. Kovács, I. Jankovics, H. Lehmann, H. Mandel, J. Peitz, D. Schäfer, W. Schmutz, J. Schweickhardt. Financial support by the Deutsche Forschungsgemeinschaft (Wo 296/20-1, 436 TSE 113/18) and the Academy of Sciences and Grant Agency of the Czech Republic (436 TSE 113/18, 202/97/0326) is appreciated.

\section{References}

Baade, D. 1990, in Angular Momentum and Mass Loss for Hot Stars, NATO ASI Series C Vol. 316, ed. L. A. Willson and R. Stalio, 177

Bjorkman, \& J.E., Cassinelli, J.P. 1993, ApJ 409, 429

Hanuschik, R.W. 1995, A\&A 295, 423

Hanuschik, R.W., Dachs, J., Baudzus, M., Thimm, G. 1993, A\&A 274, 356

Jeffery, C.S. 1991, MNRAS 249, 327

Kaufer, A. 1998, in Reviews in Modern Astronomy 11, ed. R. Schielicke, 177

Rivinius, Th., Baade, D., Štefl, S., Stahl, O., Wolf, B., Kaufer, A. 1998a, A\&A 333,125

Rivinius, Th., Baade, D., Štefl, S., Stahl, O., Wolf, B., Kaufer, A. 1998b, A\&A 336,177

Rivinius, Th., Štefl, S., Baade, D. 1999, A\&A 348, 831

Štefl, S., Baade, D., Rivinius, Th., Stahl, O., Wolf, B., Kaufer, A. 1998, in $A$ Half Century of Stellar Pulsation Interpretations, eds. P.A. Bradley and J.A. Guzik, ASP Conf. Ser., 135, 348

Sttefl, S., Hummel, W., Rivinius, Th. 2000, A\&A, 358, 208

Townsend, R.H.D.T. 1997, MNRAS, 284, 839

Zorec, J. 1994, IAU Symp. 162, 257

\section{Discussion}

A. Dudorov: What type of outburst mechanism do you consider in your numerical simulations?

S. Steff: Our model is purely empirical and it calculates the times and strengths of the outbursts from the vectorial sum of the three strongest periodicities in the 0.5- $\mathrm{d}$ group as they were derived from the observed modes and line-profile variations. The model also does not assume a physical mechanism leading to the outburst and provides - at least in its present form - no solution for the energy and angular momentum transfer. 